\title{
CYP3A5 * 3 Genetic Polymorphism is Associated with Childhood Acute Lymphoblastic Leukemia Risk: A Meta-Analysis
}

\author{
Li-Min Ma ${ }^{1}$, Hong-Chao Liu², Lin-Hai Ruan ${ }^{1}$ Yan-Ming Feng ${ }^{2}$
}

Background: Several studies have investigated the association between CYP $3 A 5 * 3$ genetic polymorphism and acute lymphoblastic leukemia (ALL) risk in children, but have yielded controversial results. Therefore, we performed a meta-analysis to evaluate synthetically the effect of $C Y P 3 A 5 * 3$ polymorphism on the risk of ALL in children.

Methods: Case-control studies investigating the relationship between $C Y P 3 A 5 * 3$ genetic polymorphism and ALL risk in children were included. Odds ratios (ORs) with 95\% confidence intervals (CIs) were calculated to assess the strength of association between CYP3A5*3 polymorphism and ALL risk in children. Q-statistic test was used to evaluate the heterogeneity and publication bias was assessed through funnel plot.

Results: In total, five case-control studies with 1070 cases and 1125 controls were included in the meta-analysis. Based on the results of heterogeneity, fixed-effects or random-effects models were applied to estimate the pooled ORs. The pooled ORs (95\% CIs) for CYP3A5*3 heterozygous mutant, homozygous mutant, and (heterozygous + homozygous) mutant were 1.47 (0.97-2.21), 1.05 (0.62-1.79),

\section{At a Glance Commentary}

Scientific background of the subject

CYP3A5 may be an important genetic contributor to inter-individual and inter-racial differences in CYP3A-mediated metabolism. The $C Y P 3 A 5 * 3$ genetic polymorphism was associated with cancer risk. However, there are conflicting findings for CYP $3 A 5 * 3$ polymorphism and the risk of children ALL.

\section{What this study adds to the field}

This meta-analysis demonstrates there is association between $C Y P 3 A 5 * 3$ polymorphism and the altered risk of children ALL, especially in Caucasian populations. These results enhance our knowledge of the childhood ALL etiology, which not only evaluate the risk of childhood ALL to protect vulnerable populations, but also will offer potential targets for therapeutics. and 1.67 (1.14-2.44) with $P=0.07,0.86$, and 0.009 , respectively. In subgroup analysis, the $Z$ values of $C Y P 3 A 5 * 3$ (heterozygous + homozygous) mutant and children with ALL in Asian and Caucasian populations were 1.34 and 2.51 with $P=0.18$ and 0.01 , respectively. No significant publication bias was detected by funnel plot.

Conclusions: The current meta-analysis showed that there was association between CYP $3 A 5 * 3$ polymorphism and the altered risk of ALL in children, especially in Caucasian populations.

(Biomed J 2015;38:428-432)

Key words: acute lymphoblastic leukemia, children, CYP3A5, meta-analysis, polymorphism

A cute lymphoblastic leukemia (ALL) is the most common cancer diagnosed in children, constituting about $30 \%$ of all childhood cancers. Although the clinical, pathological, and immunophenotypic features of this disease have been well documented, the causes of most cases of children with ALL are unknown. ${ }^{[1-3]}$ The biological mechanisms of ALL are complex and have not been fully clarified. It is generally considered that the interaction between genetic susceptibility and environmental exposures may play an important role in the etiology of ALL. ${ }^{[3-6]}$ It has been suggested that host polymorphisms may modify the individual's ability to respond to xenobiotics and maintain an intact genome in face of genotoxic stress. ${ }^{[7]}$ Thus,

From the ${ }^{1}$ Department of Hematology, the First Affiliated Hospital, Henan University of Science and Technology, Henan, China; ${ }^{2}$ Medical College, Henan University of Science and Technology, Henan, China

Received: Jun. 25, 2014; Accepted: Jan. 15, 2015

Correspondence to: Dr. Hong-Chao Liu, Medical College, Henan University of Science and Technology, Henan, China. No. 31, Anhui Rd., Jianxi District, Luoyang 471003, Henan Province, China. Tel.: 86-15036319005; Fax: 86-379-64830481; E-mail: lhongchao@ hotmail.com

DOI: $10.4103 / 2319-4170.151029$ 
polymorphisms in genes encoding xenobiotic-metabolizing enzymes may have determined susceptibility to cancer.

Xenobiotics are activated mainly by the activities of cytochrome P450 (CYP450) family of enzymes and then converted into more hydrophilic forms under the catalysis of conjugation enzymes. CYP450 enzymes are involved in the metabolism of a wide range of drugs and potential xenobiotics. The most highly expressed subfamily is CYP3A, which includes the isoforms CYP3A4, CYP3A5, CYP3A7, and CYP3A43. CYP3A5 is responsible for the metabolism of more than $50 \%$ of clinically therapeutic drugs and a variety of endogenous compounds and pro-carcinogens ${ }^{[8,9]}$ However, CYP $3 A 5$ polymorphism is more prevalent and shows significant differences in protein expression level between ethnic groups..$^{[8,10,11]}$ These variations may be due to genetic polymorphisms and by the modulation of CYP $3 A$ expression through various environmental factors and drug-drug interactions. Therefore, CYP $3 A 5$ may be an important genetic contributor to inter-individual and inter-racial differences in CYP3A-mediated metabolism. ${ }^{[8,12]}$ The most important functional single nucleotide polymorphism (SNP) in CYP3A5 gene is CYP3A5 * 3 6986A > G (rs776746). ${ }^{[13]}$ CYP $3 A 5 * 3$ confers low CYP $3 A 5$ expression because of alternative mRNA splicing. This results in the incorporation of an intron sequence into the mature mRNA, and the production of a truncated protein due to premature termination of translation. ${ }^{[8]}$ Thus, CYP $3 A 5 * 3$ genetic polymorphism may play a role in the risk of developing cancer. ${ }^{[14-16]}$

The role of $C Y P 3 A 5 * 3$ polymorphism in the development of ALL in children has been extensively investigated, but reported with inconsistent results. To investigate the possible effect of CYP3A5 variant on the ALL risk through a more robust and powered analysis, we carried out a meta-analysis of case-control studies that examined the association between CYP3A5*3 genetic polymorphism and the risk of ALL in children.

\section{METHODS}

\section{Identification and eligibility of relevant studies}

The electronic PubMed, Elsevier, EMBASE, China Biological Medicine Database (CBM), China National Knowledge Infrastructure platform (CNKI), and Wanfang database were searched for articles published before October 2013 with the following search terms: Cytochrome P4503A5 or CYP3A5; polymorphism, variant, or mutation; leukemia or acute leukemia; children, childhood, or pediatric. Literature search included all languages. References of retrieved articles were also screened to identify additional publications.

The following were the inclusion criteria used for study selection: (1) Case-control study; (2) confirmed diagnosis for the case group according to the diagnosis criteria of ALL in children; (3) genotype frequency available in both cases and controls; and (4) genotype distribution in the control group was in agreement to Hardy-Weinberg equilibrium (HWE). Abstracts, case reports, commentary, and review articles were excluded. When the case-control study was included by more than one article using the same case series, we selected the one with the largest number of participants only.

\section{Data extraction}

The following information was collected about each included study: The first author, year of publication, country of origin, ethnicity, genotyping method, and genotype distribution. Two observers extracted the information from each study independently, and disagreements were resolved by discussion and the data were evaluated by a third reviewer.

\section{Statistical analysis}

We applied odds ratios (ORs) with 95\% confidence intervals (CIs) to assess the strength of association between CYP $3 A 5 * 3$ polymorphism and ALL risk in children. The $\chi^{2}$-based Q-statistic test was used to assess between-study heterogeneity and the degree of heterogeneity was quantified with $\mathrm{I}^{2}$ metric. The result was considered significant when $p<0.05$, in which case the random-effects model (DerSimonian-Laird) was used to calculate the pooled OR. Alternatively, the pooled OR was estimated using the fixed-effects model (Mantel-Haenszel). The inverted funnel plots were used to evaluate the publication bias. HWE in the control group for all studies was checked using the $\chi^{2}$ test. All the $p$ values were two-sided, and $p<0.05$ was considered as statistically significant. All data analyses were performed with Review Manager v5.2 (The Cochrane Collaboration, Oxford, UK) and Stata v10.0 (Stata Corporation, College Station, TX, USA).

\section{RESULTS}

\section{Study characteristics}

We identified a total of 19 potentially relevant publications based on the literature search criteria. At the initial screening, nine articles were excluded; during further screening, five studies were excluded due to data duplication, data insufficiency, data error, and by assessing complication risk. Finally, five case-control studies including 1070 cases and 1125 controls met the inclusion criteria and were suitable for the meta-analysis. ${ }^{[17-21]} \mathrm{A}$ flow chart of the literature search and study selection is presented in Figure 1. The countries in which these studies had been carried out include Denmark, Thailand, China, and Brazil. The cases had a confirmed diagnosis of ALL and the controls were healthy populations matched for age, sex, and ethnicity. Genotype distribution 
among the controls of all included studies was in agreement with HWE. Detailed characteristics of the included studies are summarized in Table 1.

\section{Results of meta-analysis}

\section{Evaluation of the association between CYP3A5 *3 heterozygous mutant and ALL risk in children}

The heterogeneity test of heterozygous mutant showed $\chi^{2}=3.03, p=0.55$, indicating that there was no significant heterogeneity among these studies. The fixed-effects model was used to calculate the pooled OR and $95 \%$ CI, which were $1.47(0.97-2.21), Z=1.82$, $p=0.07$, suggesting that there was no significant association between $C Y P 3 A 5 * 3$ heterozygous mutant and ALL risk in children [Figure 2].

Evaluation of the association between CYP3A5 * 3 homozygous mutant and ALL risk in children

The heterogeneity test of homozygous mutant showed $\chi^{2}=23.82, p<0.0001, \mathrm{I}^{2}=83 \%$, indicating that there was significant heterogeneity among these studies. The random-effects model was used to calculate the pooled OR and 95\% CI, which were 1.05 (0.62-1.79), $Z=0.18$, $p=0.86$, suggesting that there was no significant association between $C Y P 3 A 5 * 3$ homozygous mutant and ALL risk in children [Figure 3].

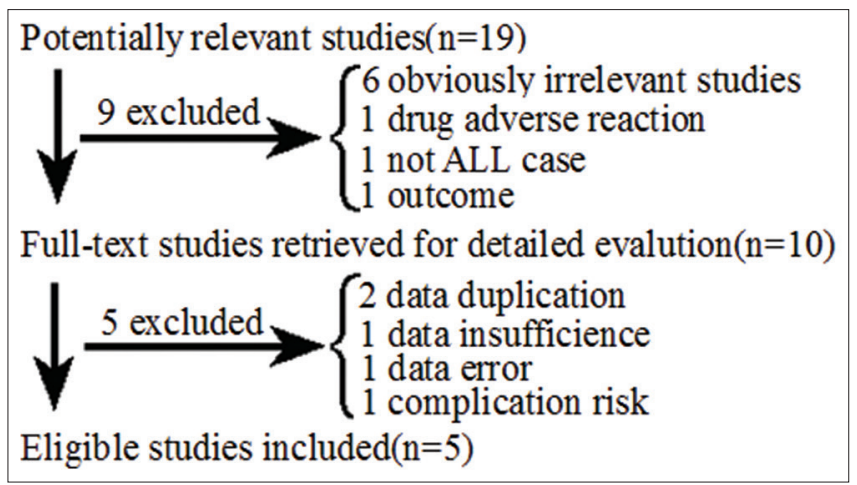

Figure 1: Flow chart of studies included in the meta-analysis.



Figure 3: Meta-analysis for the association of $C Y P 3 A 5 * 3$ homozygous mutant and ALL in children.
Evaluation of the association between CYP3A5 *3 (heterozygous + homozygous) mutant and ALL risk in children.

The heterogeneity test of (heterozygous + homozygous) mutant showed $\chi^{2}=8.57, p=0.07$, indicating that there was no significant heterogeneity among these studies. The fixed-effects model was used to calculate the pooled OR and $95 \% \mathrm{CI}$, which were 1.67 (1.14-2.44), $Z=2.63, p=0.009$, suggesting that there was significant association between CYP $3 A 5 * 3$ (heterozygous + homozygous) mutant and ALL risk in children [Figure 4].

A subgroup analysis was also performed by specific ethnicity. The results of meta-analysis of CYP3A5*3 heterozygous, homozygous, and (heterozygous + homozygous) mutant and children with ALL in Asian populations showed the values of $Z=1.02,0.01$, and 1.34 , respectively, with all $p$ values being $>0.05$ [Figures 2-4], indicating that there was no significant association between $C Y P 3 A 5 * 3$ polymorphism and the risk of ALL in children in Asian populations. Regarding Caucasians, the results gave the values $Z=1.64(p=0.10), 0.19(p=0.85)$, and $2.51(p=0.01)$, respectively, [Figures 2-4], indicating that there was significant association between CYP $3 A 5 * 3$ (heterozygous + homozygous) mutant and the development of ALL in children of Caucasian populations.

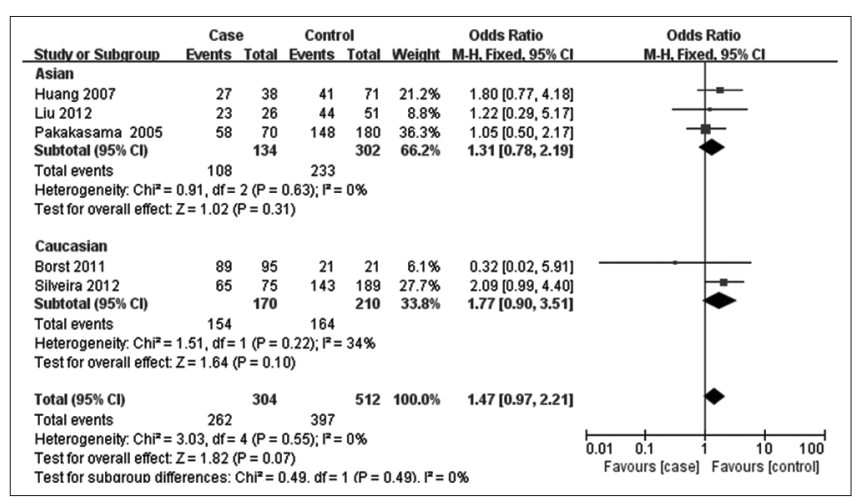

Figure 2: Meta-analysis for the association of $C Y P 3 A 5 * 3$ heterozygous mutant and ALL in children.



Figure 4: Meta-analysis for the association of $C Y P 3 A 5 * 3$ (heterozygous + homozygous) mutant and ALL in children. 
Table 1: Characteristics of the included studies of $C Y P 3 A 5 * 3$ polymorphism with ALL risk in children

\begin{tabular}{|c|c|c|c|c|c|c|c|c|c|c|c|c|c|}
\hline \multirow[t]{2}{*}{ First author } & \multirow[t]{2}{*}{ Year } & \multirow[t]{2}{*}{ Country } & \multirow[t]{2}{*}{ Ethnicity } & \multirow[t]{2}{*}{ Genotyping method } & \multicolumn{2}{|c|}{ Sample size } & \multicolumn{3}{|c|}{ Case } & \multicolumn{3}{|c|}{ Control } & \multirow[t]{2}{*}{$\overline{\text { HWE }}$} \\
\hline & & & & & Case & Control & $\mathrm{AA}$ & AG & GG & $\mathrm{AA}$ & $\mathrm{AG}$ & GG & \\
\hline Borst & 2011 & Denmark & Caucasian & TaqMan assay & 616 & 203 & 6 & 89 & 521 & 0 & 21 & 182 & Yes \\
\hline Huang & 2007 & China & Asian & PCR-RFLP & 91 & 120 & 11 & 27 & 53 & 30 & 41 & 49 & Yes \\
\hline Liu & 2012 & China & Asian & PCR-RFLP & 52 & 120 & 3 & 23 & 26 & 7 & 44 & 49 & Yes \\
\hline Pakakasama & 2005 & Thailand & Asian & PCR-RFLP & 107 & 320 & 12 & 58 & 37 & 32 & 148 & 140 & Yes \\
\hline Silveira & 2012 & Brazil & Caucasian & PCR-RFLP & 204 & 364 & 10 & 65 & 129 & 46 & 143 & 173 & Yes \\
\hline
\end{tabular}

Abbreviations: PCR-RFLP: Polymerase chain reaction-restriction fragment length polymorphism; HWE: Hardy-Weinberg equilibrium

\section{Publication bias and sensitivity analysis}

Publication bias was assessed through funnel plot, which provides a visual sense of the relationship between effect size and precision for publication bias among the studies included in the meta-analysis. Results showed that all points in the funnel plots were symmetrically distributed, suggesting that no significant bias was detected by funnel plot. Sensitivity analysis, in which the pooled ORs were calculated after omission of one study with the smallest sample size, revealed that the combined estimates remained virtually the same, suggesting the robustness of our results (data not shown).

\section{DISCUSSION}

A variety of factors might be involved in the development of ALL, including chemical substances, physical factors, and exposure to virus. ${ }^{[3,22]}$ Molecular epidemiological studies have suggested that genetic polymorphisms may play an important role in the etiology of ALL, and genetic variations of xenobiotic-metabolizing enzymes have been proved to influence the risk of this disease..$^{[2-4]}$ The human CYP3A5 gene is located on chromosome 7q21.1 and spans approximately $32 \mathrm{~kb}$ in length. ${ }^{[23]} \mathrm{CYP} 3 \mathrm{~A} 5$ is a member of the CYP3A subfamily which metabolizes approximately $50 \%$ of therapeutic drugs, steroid hormones, and extensive xenobiotics. ${ }^{[8]}$ CYP $3 A 5$ genetic polymorphisms exhibit inter-individual differences in CYP3A5 expression. So far, at least 34 SNPs of CYP3A5 gene have been identified. ${ }^{[24]}$ Among them, the most frequent and functional polymorphism is the $\mathrm{A}$ to $\mathrm{G}$ transition in intron 3 , which has been named $C Y P 3 A 5 * 3$ polymorphism. The $C Y P 3 A 5 * 3$ allele results in a truncated protein with loss of $C Y P 3 A 5$ expression and enzyme activity. Individuals carrying $C Y P 3 A 5 * 3$ allele show slower than average metabolism of CYP3A substrates. Remarkable inter-ethnic variations in the frequencies of alleles and genotypes for the CYP3A5*3 polymorphism have been reported, for instance, the CYP $3 A 5 * 3$ variant allele is more frequent in Dutch Caucasians (91.7\%) than in Chinese (57.9\%) and is much less common in African Americans (47.5\%). ${ }^{[8,10,19,24]}$ It is suggested that the CYP $3 A 5$ is an important genetic contributor to inter-individual dif- ferences in CYP3A-dependent metabolism. Therefore, the CYP $3 A 5 * 3$ genetic polymorphism is associated with cancer risk. However, there are conflicting findings for $C Y P 3 A 5 * 3$ polymorphism and the risk of ALL in children. Borst et al. investigated the impact of the CYP $3 A 5 * 3$ variant on the risk of childhood ALL and demonstrated an increased risk to develop the disease compared to the wild allele. ${ }^{[17]}$ In contrast, Pakakasama et al. did not detect significant differences of genotype or allele frequencies for CYP3A $5 * 3$ polymorphism between ALL and control groups. ${ }^{[21]}$

In the current meta-analysis, we examined the possible association between CYP3A5 * 3 polymorphism and ALL risk in children. The results we obtained were that the pooled ORs with 95\% CIs of CYP3A5*3 heterozygous mutant, homozygous mutant, and (heterozygous + homozygous) mutant were $1.47(0.97-2.21), 1.05(0.62-1.79)$, and 1.67 (1.14-2.44) with $p=0.07,0.86$, and 0.009 , respectively, indicating that there was significant association between CYP3A5 * 3 (heterozygous + homozygous) mutant and the risk of ALL in children. The results of subgroup analysis showed that the $C Y P 3 A 5 * 3$ (heterozygous + homozygous) mutant was associated with the development of ALL in Caucasian children $(\mathrm{Z}=2.51, p=0.01)$, but not with the risk of ALL in Asian children $(Z=1.34, p=0.18)$. No significant publication bias was detected by funnel plot, and sensitivity analysis suggested the robustness of present results.

\section{Conclusions}

In summary, our meta-analysis showed that there was association between CYP $3 A 5 * 3$ polymorphism and the altered risk of ALL in children, especially in Caucasian populations. These comprehensive results deeply enhance our knowledge of the etiology of childhood ALL, which not only evaluate the risk of childhood ALL in order to protect vulnerable populations but also offer potential targets for therapeutics. However, since limited sample cases were included in this study, and selection bias as well as potential confounders (e.g., sex, ethnicity, and exposures) might influence the combined results, studies with larger sample sizes and better study designs are required in future to confirm the findings of the present study, providing further insights into the etiology and targeted prevention of ALL in children. 


\section{Financial support and sponsorship}

Nil.

\section{Conflicts of interest}

There are no conflicts of interest.

\section{REFERENCES}

1. Pui CH, Evans WE. Treatment of acute lymphoblastic leukemia. N Engl J Med 2006;354:166-78.

2. Wiemels J. Perspectives on the causes of childhood leukemia. Chem Biol Interact 2012;196:59-67.

3. Inaba $\mathrm{H}$, Greaves M, Mullighan CG. Acute lymphoblastic leukaemia. Lancet 2013;381:1943-55.

4. Belson M, Kingsley B, Holmes A. Risk factors for acute leukemia in children: A review. Environ Health Perspect 2007;115:138-45.

5. Buffler PA, Kwan ML, Reynolds P, Urayama KY. Environmental and genetic risk factors for childhood leukemia: Appraising the evidence. Cancer Invest 2005;23:60-75.

6. Chokkalingam AP, Bartley K, Wiemels JL, Metayer C, Barcellos LF, Hansen HM, et al. Haplotypes of DNA repair and cell cycle control genes, X-ray exposure, and risk of childhood acute lymphoblastic leukemia. Cancer Causes Control 2011;22:1721-30.

7. Anderson LM. Environmental genotoxicants/carcinogens and childhood cancer: Bridgeable gaps in scientific knowledge. Mutat Res 2006;608:136-56.

8. Kuehl P, Zhang J, Lin Y, Lamba J, Assem M, Schuetz J, et al. Sequence diversity in CYP3A promoters and characterization of the genetic basis of polymorphic CYP3A5 expression. Nat Genet 2001;27:383-91.

9. Paulussen A, Lavrijsen K, Bohets H, Hendrickx J, Verhasselt P, Luyten $\mathrm{W}$, et al. Two linked mutations in transcriptional regulatory elements of the CYP3A5 gene constitute the major genetic determinant of polymorphic activity in humans. Pharmacogenetics 2000;10:415-24.

10. Bains RK, Kovacevic M, Plaster CA, Tarekegn A, Bekele E, Bradman NN, et al. Molecular diversity and population structure at the Cytochrome P450 3A5 gene in Africa. BMC Genet 2013;14:34.

11. Lai Y, Zhang J, Wang YX, Wang XD, Li JL, Wang YH, et al. CYP3A5 * 3 and MDR-1 C3435T single nucleotide polymorphisms in six Chinese ethnic groups. Pharmazie 2011;66:136-40.

12. Shirasaka Y, Chang SY, Grubb MF, Peng CC, Thummel KE, Isoherranen $\mathrm{N}$, et al. Effect of CYP3A5 expression on the inhibition of CYP3A-catalyzed drug metabolism: Impact on modeling CYP3A-mediated drug-drug interactions. Drug Metab Dispos 2013;41:1566-74.
13. Lamba JK, Lin YS, Schuetz EG, Thummel KE. Genetic contribution to variable human CYP3A-mediated metabolism. Adv Drug Deliv Rev 2002;54:1271-94.

14. Feng WX, Liu F, Gu Y, Jiao WW, Sun L, Xiao J, et al. Functional polymorphisms in CYP2C19 and CYP3A5 genes associated with decreased susceptibility for paediatric tuberculosis. Indian J Med Res 2012;135:642-9.

15. Hyland PL, Freedman ND, Hu N, Tang ZZ, Wang L, Wang C, et al. Genetic variants in sex hormone metabolic pathway genes and risk of esophageal squamous cell carcinoma. Carcinogenesis $2013 ; 34: 1062-8$

16. Sailaja K, Rao DN, Rao DR, Vishnupriya S. Analysis of CYP3A5*3 and CYP3A5 * 6 gene polymorphisms in Indian chronic myeloid leukemia patients. Asian Pac J Cancer Prev 2010;11:781-4.

17. Borst L, Wallerek S, Dalhoff K, Rasmussen KK, Wesenberg F, Wehner PS, et al. The impact of CYP3A $5 * 3$ on risk and prognosis in childhood acute lymphoblastic leukemia. Eur J Haematol $2011 ; 86: 477-83$.

18. Silveira VS, Canalle R, Scrideli CA, Queiroz RG, Lopes LF, Tone LG. CYP3A5 and NAT2 gene polymorphisms: Role in childhood acute lymphoblastic leukemia risk and treatment outcome. Mol Cell Biochem 2012;364:217-23.

19. Huang Z. Significance of the expression of the CYP3A5 genetic polymorphism in childhood acute leukemia. Suzhou, Jiangsu, China: Suzhou University; 2007. (In Chinese).

20. Liu Y, Lu XT, Sheng Q, Tian HP, Xu AJ, Tang YN, et al. Association of CYP3A 5 * 3 with Acute Lymphoblastic Leukemia susceptiveness in Chinese Han children. Chin J Clin Pharmacol Ther 2012;17:73-7.

21. Pakakasama S, Mukda E, Sasanakul W, Kadegasem P, Udomsubpayakul U, Thithapandha A, et al. Polymorphisms of drug-metabolizing enzymes and risk of childhood acute lymphoblastic leukemia. Am J Hematol 2005;79:202-5.

22. Scélo G, Metayer C, Zhang L, Wiemels JL, Aldrich MC, Selvin S, et al. Household exposure to paint and petroleum solvents, chromosomal translocations, and the risk of childhood leukemia. Environ Health Perspect 2009;117:133-9.

23. Gellner K, Eiselt R, Hustert E, Arnold H, Koch I, Haberl M, et al. Genomic organization of the human CYP3A locus: Identification of a new, inducible CYP3A gene. Pharmacogenetics 2001;11:111-21.

24. Lee SJ, Usmani KA, Chanas B, Ghanayem B, Xi T, Hodgson E, et al. Genetic findings and functional studies of human CYP3A5 single nucleotide polymorphisms in different ethnic groups. Pharmacogenetics 2003;13:461-72.

This is an open access article distributed under the terms of the Creative Commons Attribution-NonCommercial-ShareAlike 3.0 License, which allows others to remix, tweak, and build upon the work non-commercially, as long as the author is credited and the new creations are licensed under the identical terms.

For reprints contact: reprints@medknow.com 